$\xi=1$ 国

\title{
An End To End Ioe-Based Technology Solution for Diabetic Healthcare
}

\author{
Mustafa Abdulkadhim ${ }^{1}$ \\ ${ }^{1}$ Assist; Lecturer in AL-Nahrain university, college of information engineering \\ *Corresponding author E-mail : Mustafa.kadhim@coie-nahrain.edu.iq
}

\begin{abstract}
In the context of keeping the elderly healthy and safe and with the development of the modern small energy efficient technology, with the help of a health monitoring device (HM) as a main activity. The Cloud-based IoT architecture contains a cloud computing and the Internet of Things In order to focus on the advantages that was derived from the complementarities. The sensing services is focused on the development of the sensors data usage and accessibility. beginning from the general scenarios of this service, this paper discusses a Human Machine-oriented Sensing Service scenario with the goal to make the quality of the service and its implementation, taking In consideration the demanding interests of this service which doesn't come only from big medical facilities but also from general practitioners and small clinics. A mathematical model for simulating induced hyperglycemia and hypoglycemia and a smartwatch application is implemented for the patient as well as the health monitoring company (HMC) and the cloud infrastructure for the data to be stored was designed as well as the infrastructure necessary for the patient to run the solution in his/her home.
\end{abstract}

Keywords: real-time Health monitoring; IoT Cloud computing; Sensing services

\section{Introduction}

in most major of the developed countries - according to WHO -, the percentage of over-seventy aged population is increased rapidly and the total number of the people aged 70 or more will be doubled within 2026. An increasing aging society will also mean an increase in the percentage of the people who may need either a lifetime medical observation or a constant help to carry on their daily life. Internationally known institutions and national governments are, therefore, suffering to find new cheap ways to care about the elderly, also to take care of the patients suffering from diabetes and patients with heart problems, in order to make the patients depend less on private clinics and hospitals. A new approach instead is created using electronic health solutions. A new idea on the great expectations behind E-Health can be done by keeping an eye on the WHO on this matter and by examining the surveys about the implementation of electronic-Health systems and various applications. A very recent emerged technology the Internet of Things, will have the power to determine new methods for evolution in electronic-health systems. In fact, IoT devices are capable of bringing the smart devices -that can be connected to the cloud and can be addressed uniquely- closer to the proximity of the patients

In spite of the impressive potential of IoT that were mentioned previously, a practical full implementation of E-health systems and IoT are still far away from the truth. On one hand Old Ehealth application is implemented using fixed devices that are known to be installed purposely during system deployment. On the other hand, when using IoT the system will be constructed by a huge number of devices that will be smart and create an assisted environment for the patient. Also, all the mentioned things might be implemented by an organization or a company for other reasons than assisting suffering people. [1]. Therefore, any E-health system must have these management features in order to be integrated with IoT: (i) to automatically and dynamically be able to locate things within parameter of the patient, (ii) be able to select individuals that can carry out a specific task, and (iii) to assess correctly their trustworthiness as researched and contributed by the SIoT model [2]. SIoT might be the answer to the personal related management problems, according to their research it has proven the ability to foster the service discovery, source crowding and resource visibility in its context. That said these studies still at the beginning concerning the full implementation of the SIoT paradigm. The contribution of this paper is of twofold and consists of:

- designing a specific model for the electronic-Health and the IoT integration, that will rely on the SIoT model.

- measuring the performance, achievability and the benefits using social-awareness approach for devices in the E-health IoT based system.

The rest of this paper is prepared as follows. Section II will present the motivation and the related work for e-health IoT systems integration so far. Section III presents the designed cloud-based framework - based on the SIoT paradigm - for IoT and E-health, Section IV presents an implemented mathematical model that was implemented to calculate a hyperglycemia and hypoglycemia cases. Section V gives the implemented E-Health IoT model, and section VI provides a quantitative assessment and will evaluate the contribution. And finally, the conclusion will be presented in section VII. 


\section{Motivation and Related Work.}

As of later, there have been rapid improvements in observing innovation for free living, early treatment administrations and diagnosis availability. In this domain, an overview of these advances, alongside their potential, is given.

L. Atzori et al in his paper discussed a new SIoT paradigm was implemented and designed, in which it uses people and devices to collect sensor data and cloud-based service to analyze it [2].

Gabriel Neagu et al, discussed a human - machine (HM) design approach in which the interaction between the two was discussed. [1].

Giuseppe Ruggeri et al, developed a new paradigm that was built on top of the SIoT and it was evaluated and performed [3].

Yesenia Cruz Rosado et al discussed a new mathematical implementation for the glucose calculation for diabetes type- 1 and type$2[4]$.

M. Mazhar et al, used an IoT body area network (BAN) to collect sensor data in real tam and analyze these data using Hadoop and big data analytics approach in order to deal more efficiently with these medical important data. [5]

\section{Cloud-Based Health Monitoring-Based Iot Model}

\subsection{System Architecture Philosophy:}

CLOUD-structured IoT is a combination between IoT and cloud infrastructure, and it is important for many reasons: storage and computational capabilities both limited or not, components that are used (virtual Vs real resources), also ubiquitous vs limited coverage and the usage of the internet (service delivery Vs data transmission). Also, it was relevant to see these complimentary to generate useful advantages if the integration was the thing to consider: the resources used by the cloud and their scalability in deployment will cover for IoT technology constrains, while the interactivity and ease of implementation of IoT technology and its interactivity with the real world enabled many of these cloudbased applications to run [6]. With respect to HM specification, the dynamic nature of the data made it more convenient to use cloud-based services, regardless of the place or time for accessing the data of the patient, also the pay per usage feature will make it cost effective to use the system other than traditional health services combining with that data recovery if the equipment got damaged once the data stored and replicated on the cloud it can be easily recovered when needed. Also, this integration of cloud computing and IoT technology in health applications can improve the life situation for the elderly by providing them with remote monitoring and personalized healthcare and reminders with standby medical units to respond immediately and actively in Realtime in case of an emergency especially for those elderly with chronic diseases like diabetes and heart problems [1].

\subsection{The Architectural Solution}

In the proposed End to End IoE-Based Technology Solution the following components are interconnected using a cloud-based computing platform: [7]. a medical infrastructure (the hospital), an emergency, a personal home and environment to the patient, their family. The designed architecture of the system proposed in this paper is constructed of three different layers: (1) the data aggregation layer (a raw data that is heterogenous in their formats and structure that to be collected and gathered from many sources and then processed previous to the transmission to the next layer), (2) a layer to manage the data (a heterogeneous distributed data processing, analysis and a data storage capabilities), and (3) an application service layer. The hospital networking architecture that locates, monitors and detects objects consists of 6 layers : data conversion layer (to collect the data using sensors near the patients), data synthesis layer (that translated and stores data in the database), cloud based layer (that will make the resources available via the cloud and many other services like recovery and alerting), a network layer (that will route the data through different nodes from a source to a destination), a reasoning knowledge layer (a decision making support for data mining purposes), and a visualization-based layer (data re-representation for the purpose of community reading availability). The Cloud Things-based model is oriented on developing, operating, deploying and composing IoT-based applications using cloud platform.

\subsection{Iot-Based Applications in H-M (Human Machine)}

There are many differences of $\mathrm{H}-\mathrm{M}$ implementations based on internet of things model. Some examples are

diabetes (using opto-physiologic that is a non-invasive; and then convert the analog signal to a digital one), heart-rate monitoring device (a wireless transmitter and electrodes), BP monitoring device (automatically inflating and wearable sensor that senses BP and send it wirelessly), a temperature monitoring probe (a small sensor that senses the skin and body temperature and report it back wirelessly) [8]. rehab monitoring device (event tracking and reporting also wearable and wirelessly transmitting patient's data), a cough analysis device (audio-based system; that records and analyzes spectrograms), melanoma analysis application (a camerabased application in the smartphone based on image processing to compare images taken by the patient with a stored library of cancerous images) [9].

\section{The Mathematical-based Model}

The results collected form the patient's Glucose tolerance test GTT are needed to be interpreted for diabetic patients, therefore a mathematical model is proposed here in order to interpret these tests. Also, the glucose levels play an important and essential rule in the performance of the model. Glucose levels depends on different hormones like epinephrine and insulin and many other hormones like glucagon and thyroxine. The proposed model here will be separated into three different groups according to the hormones which affects the levels of glucose in blood. soy we can isolate all hormones that rises glucose levels in the from those hormones that can lower the glucose levels in blood [4].

The variables:

$$
\begin{aligned}
& \left.\frac{\mathrm{d} F(t)}{\mathrm{d} t}=G_{1}\left(F(t)_{0} E(t)_{0} P(t)\right)+K(t)\right) \\
& \frac{d E(t)}{d t}=G_{2}\left(F(t)_{0} E(t)_{0} P(t)\right) \\
& \frac{d P(t)}{d t}=G_{3}\left(F(t)_{0} E(t)_{0} P(t)\right)+J(t)
\end{aligned}
$$

In which $K(t), J(t)$ denote the outside rates of the supplied hormones and glucose (such as insulin). As it is, this model is fairly general. A small deviation from steady state will be considered. If $(F, P, E)$ represents that state, then will be modelled by the equations:

$G_{1}\left(F_{0}, E_{0}, P_{0}\right)=0, G_{2}\left(F_{0}, E_{0}, P_{0}\right)=0, G_{3}\left(F_{0}, E_{0}, P_{0}\right)=0$.

also, we assume that $\mathrm{F}, \mathrm{E}$ and $\mathrm{P}$ have also accomplished their optimal situation F0, E0 and P0 by the time that the fasting patient has arrived to a hospital. Let 
$f(t)=F(t)-F_{0}, \quad e(t)=E(t)-E_{0}, \quad h(t)=P(t)-P_{0}$

represent very small deviation from the optimal situation. therefore, we have linearized equations (1), (2) and (3):

$\frac{d f(t)}{d t}=x_{1} f(t)+y_{1} e(t)+\mathrm{z} P(t)+J(t)$

$\frac{d e(t)}{d t}=x_{2} f(t)+y_{2} e(t)+z_{2} P(t)$

$\frac{d P(t)}{d t}=x_{3} f(t)+y_{3} e(t)+z_{3} p(t)+K(t)$

Notice that adrenaline, growth hormone and glucagon the are those hormones that rises blood glucose, and that insulin and thyroxin have the reverse effect. These considerations will enable us to calculate the signs of these coefficients for the system. For example: if the glucose levels are higher than zero ( $f>0$ ) and the level of other hormones and the glucagon equal to zero (or low) $(\mathrm{e}=0, \mathrm{p}=0)$, then the glucose levels should be decreased $(\mathrm{a} 1<0)$ according to tissues absorption. When the glucagon levels are greater than zero or high $(\mathrm{e}>0)$ and the glucose and the other hormones levels equal to zero (or low) $(\mathrm{f}=0, \mathrm{p}=0)$, then the levels of glucose should be increased ( $b 1>0$ ) and because of the process that the glycogen turning into glucose. A Similar consideration led us to the following inequalities:

$x_{1}<0, y_{1}>0, z_{1}<0, x_{2}<0, y_{2}<0, z_{2}>0, x_{3}>0, y_{3}>0, z_{3}<0$

This sign can be included explicitly into this system for equations (6), (7) and (8) by re-writing it as shows below:

$f^{\prime}(t)=-\alpha_{1} f(t)+\beta_{1} e(t)-\gamma_{1} p(t)+J(t)$

$e^{\prime}(t)=-\alpha_{2} f(t)-\beta_{2} e(t)+\gamma_{2} p(t)$

$p^{\prime}(t)=\alpha_{3} f(t)+\beta_{3} e(t)-\gamma_{3} p(t)+K(t)$

Keeping in mind that the constants $\alpha \mathrm{i}, \beta \mathrm{i}, \gamma \mathrm{i}$, are positive numbers.

\section{The Implementation Model}

Let's suppose that mustafa is a 60 -year-old type one diabetic who is living by himself. John has always been in trouble to keep his glucose levels within healthy ranges. The proposed healthcare IoE solution will enable mustafa to always monitor his important health indicators and his condition and to call help if necessary. mustafa will have to wear a smart device to monitor the exercise levels and respiration. mustafa will also wear (CGM) which is a Continuous Glucose Monitor that will report glucose levels readings. data collected from devices will then be relayed to the Health Monitoring corporation (HMC), which will in its part deliver medical assistance in case the patient's condition become critical.

The medical staff will be in the emergency mobile treatment center (EMTC) and can monitor the patient levels while transporting to his house for medical response.

\subsection{The health monitoring company (HMC)}

The health monitoring company architecture was designed and configured as show below:

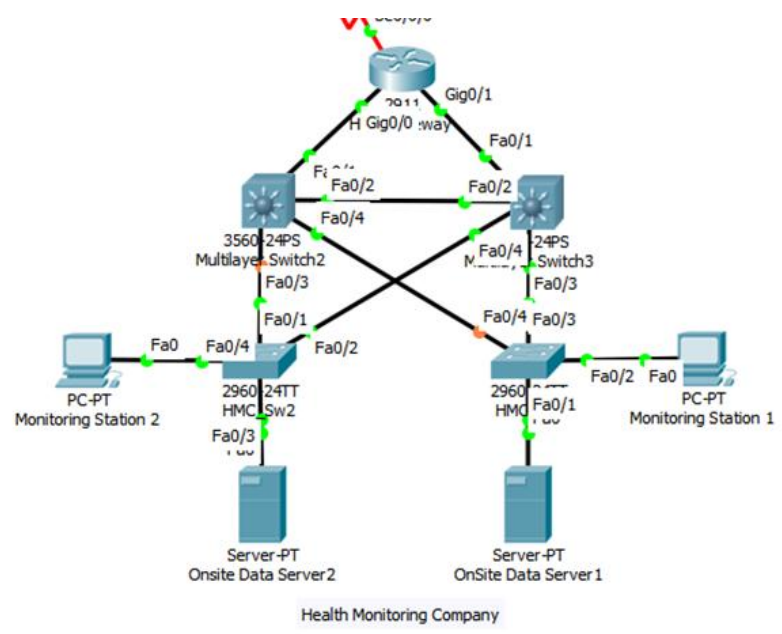

Fig.1: the health monitoring company architectural design

From above we can see that we have redundancy to the used storage as we as monitoring and networking devices, on-site servers are storing the data and a monitoring stations can be used to monitor in real time the patients' health status.

\subsection{The Cloud Data Storage Service}

The architecture of the cloud monitoring services is as shown below:

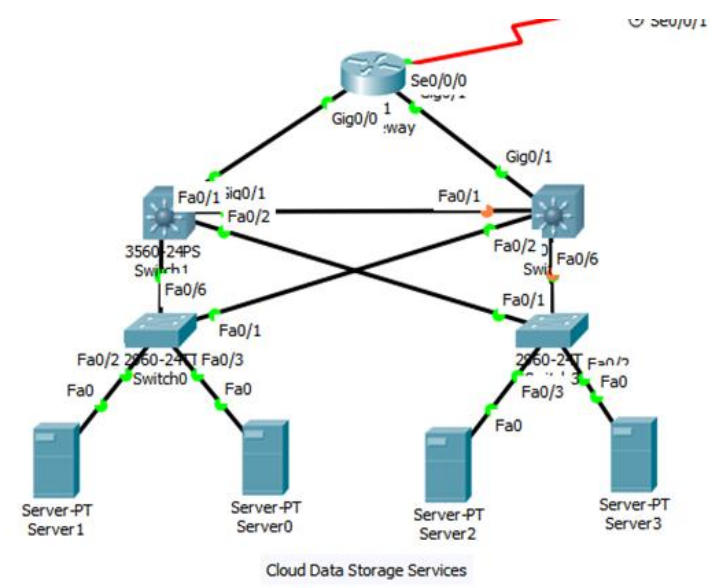

Fig.2: the cloud data storage architectural design

The proposed architecture shows that full redundancy is being used to store the patient's data in case of a sever failure full information retrieval can be obtained.

\subsection{The Emergency Mobile Treatment Center}

The mobile treatment center will access the patents data through a wireless link and can monitor in Realtime the status of the patient which will better prepare the medical staff for the treatment of the patient once arrived to the patient's home [10]. The emergency mobile treatment center will be an ambulance equipped with a monitoring station that is connected to the company servers which will enable the staff to monitor the patient condition.

\subsection{Patients Home:}

The patient home will link the glucose monitoring device as well as the respiratory monitoring device using the wireless home router to the internet to send the data (which is the respiratory data and the glucose levels) to the health monitoring company and the 
cloud service site and the mobile treatment center [11]. the proposed home architecture is show in fig (4) ad (5) below:

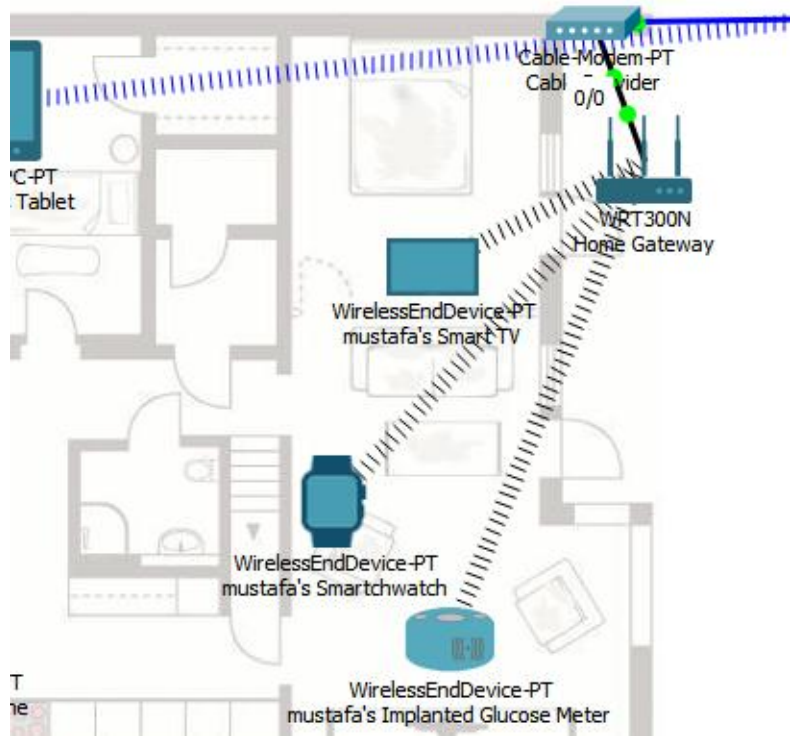

Fig.3: patient's home setup

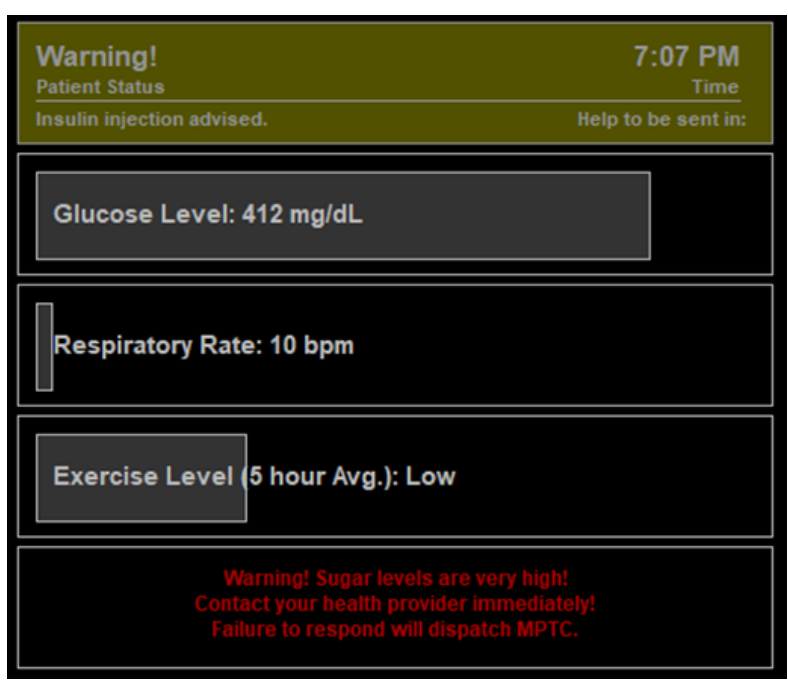

Fig.4: the application reading an induced hyperglycemia situation from the patient's sensor

\section{Results and Evaluation}

To evaluate the scalability and how it will affect the performance we increased the no. of sensors up to 25 sensors and we evaluated the average processing time of the system, we can notice that the system will each a steady state $2.8 \mathrm{msec}$. as an average processing time with the corresponding used no, of sensors.

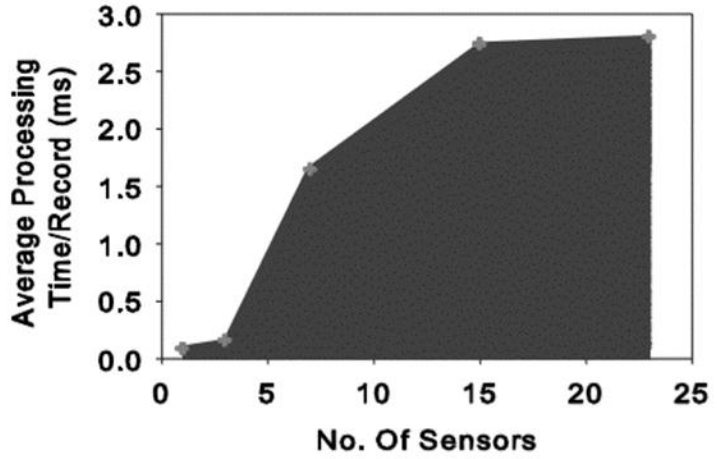

Fig.5. system efficiency with respect to the number of sensors per second.
In the next performance evaluation, we can notice that within the increase of the no. of sensors the increase happened in the dataset size which resulted in an increase in the throughput of the system. As we can see from the results in fig (5). This is due to the efficiency and redundancy that is done in the network in both the cloud and the data centers in which led to a better system utilization.

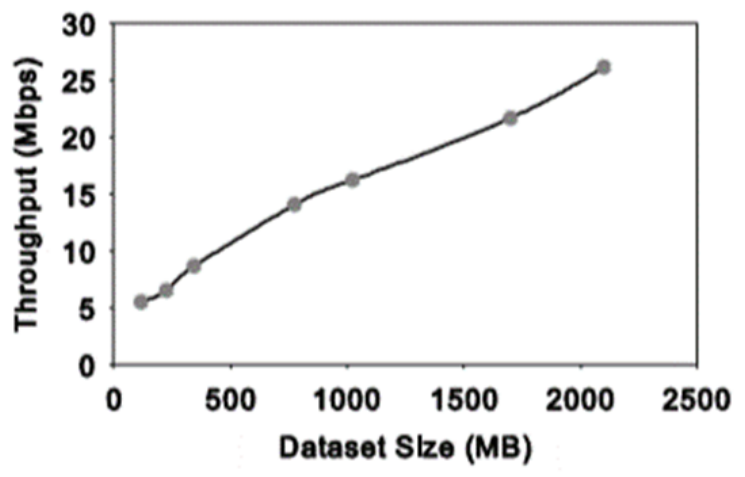

Fig.6. system throughout with respect to an increase in the dataset.

\section{Conclusion}

In the proposed end-to-end health monitoring IoE based solution we can come to a conclusion that although this still to be a future hope to be implemented in the real life it still a very viable and efficient solution which is based on a technology that exists in these days. The main challenge was how to calculate the patient correct glucose levels and that was solved using an accurate and tested mathematical model. Also, the system performance was tested and evaluated in terms of throughput and average processing time.

\section{References}

[1] G. Neagu and Ș. Preda, "A Cloud-IoT Based Sensing Service for Health Monitoring," in 6th IEEE International Conference on EHealth and Bioengineering, Sinaia, Romania, 2017.

[2] L. Atzori and A. Iera, "The Social Internet of Things (SIoT) When social networks meet the Internet of Things: Concept, architecture and network characterization," Computer Networks, vol. 56, no. 16, p. $3594-3608,2012$.

[3] G. Ruggeri and O. Briante , "A framework for IoT and E-Health Systems Integration based on the Social Internet of Things Paradigm," in 2017 International Symposium on Wireless Communication Systems (ISWCS), Bologna, 2017.

[4] Y. Cruz , "Mathematical Model for Detecting Diabetes," in National Conference On Undergraduate Research (NCUR) , Wisconsin , 2009.

[5] M. Rathore and A. Ahmad, "Real-time Medical Emergency Response System: Exploiting IoT and Big Data for Public Health," in TRANSACTIONAL PROCESSING SYSTEMS, New York , 2016.

[6] M. Abdulkadhim, "Routing Protocols Convergence Activity and Protocols Related Traffic Simulation With It's Impact on the Network," International Journal of Science, Engineering and Computer Technology, vol. 5, no. 3, p. 40, 2015.

[7] M. Abdulkadhim, "Security measures effect over performance in service provider network," Journal of Fundamental and Applied Sciences, vol. 10, no. 4s, pp. 677-681, 2018.

[8] M. A. Neamah, "Service Performance Evaluation for WiMAX Networks Based on Node Trajectory," International Journal of Soft Computing and Engineering, vol. 5, no. 2, pp. 1-3, 2015.

[9] M. Park, H. Kim and H. Ahn , "An IoT-aware workflow model designer for smart alarm service about companion animal health check," in 2018 20th International Conference on Advanced Communication Technology (ICACT), Chuncheon-si Gangwon-do, 2018.

[10] M. Abdulkadhim and K. Korabu, "Future System: Using Manet in Smartphones the Idea the Motivation and the Simulation," in 
Computational Intelligence and Information Technology, Berlin, Heidelberg, Springer, 2011, pp. 716-721.

[11] M. A. Neamah, "Design and Implementation of a Wireless Sensor Networks Application Framework," International Journal of Computer Applications, vol. 114, no. 19, pp. 41-43, 2015.

[12] s. vitale and v. vidhat, "Hybrid data-centric routing protocol of wireless body area network," in International Conference on Advances in Computing, Communication and Control (ICAC3), Mumbai,, 2017.

[13] and G. Raj , "IoT-based real-time poultry monitoring and health status identification,," in 2018 11th International Symposium on Mechatronics and its Applications (ISMA), sharjah, 2018.

[14] M. A. and K. Mahmud, "A complete Internet of Things (IoT) platform for Structural Health Monitoring (SHM)," in 2018 IEEE 4th World Forum on Internet of Things (WF-IoT),, Sigapore, 2018.

[15] M. Almulhim and N. Zaman, "Proposing secure and lightweigh authentication scheme for IoT based E-health applications,", in 2018 20th International Conference on Advanced Communication Technology (ICACT), Chuncheon-si Gangwon-do, Chuncheon-si Gangwon-do, 2018.

[16] H. Nath , "Health Monitoring using Internet of Things (IoT)," in Industrial Automation and Electromechanical Engineering Conference (IEMECON), 2017 8th Annual, Bangkok, 2017.

[17] Y. Ould-Yahia, "Towards privacy and ownership preserving of outsourced health data in IoT-cloud context," in 2018 International Symposium on Programming and Systems, Algiers, 2018. 